\title{
Hierarchical and Cooperative Model Predictive Control of Electrical Grids by using Overlapping Information
}

\author{
Víctor Sanz i López ${ }^{1}$ and Toru Namerikawa ${ }^{2}$
}

\begin{abstract}
The presented study deals with hierarchical and cooperative model predictive control (MPC) of electrical grids. The aim of this study is minimizing electrical frequency deviation while ensuring power levels do not rise too much. The original system is a simply interconnected one divided in several areas and, in order to control eventually disconnected areas due to communication blackouts, an expansion of the original system to a hierarchical version of itself by overlapping original system's areas is developed.
\end{abstract}

\section{INTRODUCTION}

Smart grids are seen as the solution to deal with future challenges in the field of electrical generation, transport and supply. As it is said in [1], environmental, economic and social issues have encouraged distributed generation and new control methods should be applied to ensure grid frequency deviation to be low enough in order to prevent potential damage to devices connected to the electrical grid. At the same time, supply cost and power should be controlled to avoid exceeding certain margins. Furthermore, the until now classical generation structure based on large power plants as the main supply sources for urban areas is changing to a set of smaller generation distributed points. To adapt to this change in terms of generation model, studies such as [2] and have been presented [3], proposing model predictive control methods (MPC) in its various versions.

A distributed control is proposed in this study, where all areas have an influence on neighbour areas. From the MPC versions enlisted in [4], the method presented is a hierarchical cooperative model predictive control of electrical grids (one of the MPC types from the ones presented in [5]), based on [6] and [7], for controlling grid's frequency. The procedure consists in expanding the original system to its hierarchical version, overlapping some subsystems, based on the formulation in [8], [9] and [10]. Model predictive control (MPC) is applied to the hierarchical system and after that the inverse way is taken to recover the original non-hierarchical system. This corresponds to the load frequency control (LFC) and the reference values come from the economic dispatch (ELD) optimization problem presented in [6]. The hierarchical cooperative control structure of the new system ensures the control of all areas in case of a hypothetical communication blackout.

\footnotetext{
*This work was supported by the partnership between Keio University and UPC-ETSEIB

${ }^{1}$ V. Sanz i López is PhD student at Institut de Robòtica i Informàtica Industrial, CSIC-UPC (Universitat Politècnica de Catalunya in Barcelona, Catalonia, Spain). vsanzeiri.upc.edu

${ }^{2} \mathrm{~T}$. Namerikawa is professor in Keio University at the Department of System Design Engineering, Graduate School of Science and Technology, Yokohama, Kanagawa, Japan. namerikawa@sd.keio.ac.jp
}

\section{PROBLEM FORMULATION}

The system studied is an interconnected electrical grid composed by several interconnected areas, which can be seen in Fig. 1 and is referred as $S$. Each area has the structure presented in diagram from Fig. 2.

\section{A. State-space model for each area}

This so called system $S$ can be divided into several areas $i$ whose state-space representation can be expressed as follows.

$$
\begin{aligned}
x_{i}(k+1) & =\sum_{j=1}^{n} A_{i j} x_{j}(k)+B_{i} u_{i}(k)+H_{i} w_{i}(k) \\
z_{i}(k) & =C_{i} x_{i}(k)+D_{i} u_{i}(k)
\end{aligned}
$$

System variables and matrices presented in equations (1) and (2) are state variables $x_{i}(k) \in \mathbb{R}^{n_{1}}$, input variables $u_{i}(k) \in$ $\mathbb{R}^{m_{1}}$, disturbance variables $w_{i}(k) \in \mathbb{R}^{q_{1}}$ and output variables $z_{i}(k) \in \mathbb{R}^{p_{1}} . A_{i i} \in \mathbb{R}^{n_{1} \times n_{1}}, B_{i} \in \mathbb{R}^{n_{1} \times m_{1}}, C_{i} \in \mathbb{R}^{p_{1} \times n_{1}}$, $D_{i} \in \mathbb{R}^{p_{1} \times m_{1}}$ and $H_{i} \in \mathbb{R}^{n_{1} \times q_{1}}$ are matrices in equations (1) and (2). Matrices $C_{i}$ and $D_{i}$ are positive definite matrices.

\section{B. State-space model for the overall system}

Given the state-space representation for each area $i$ the global state-space representation can be defined. To do so a new variable $v_{i}(k)=\sum_{j=2, j \neq i}^{n} A_{i j} x_{j}(k)+H_{i} w_{i}(k)$ is defined ( $n$ is the number of areas) and the following statespace representation is presented.

$$
\begin{gathered}
x(k+1)=A_{D} x(k)+B u(k)+v(k) \\
z(k)=C x(k)+D u(k)
\end{gathered}
$$

Fig. 1: Electrical power network [9]

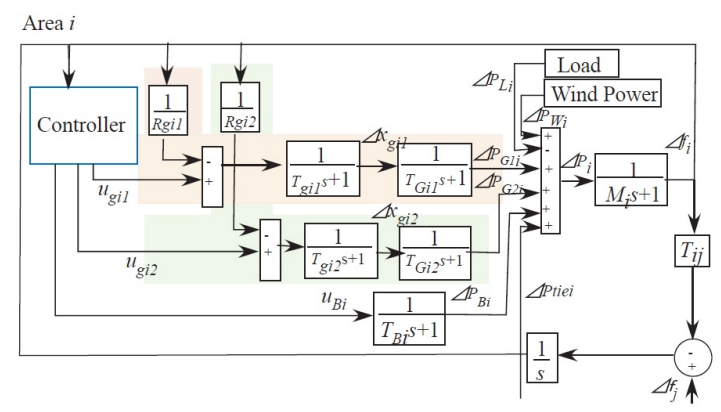

Fig. 2: Area $i$ model 
Where,

$$
\begin{array}{r}
x(k)=\left[x_{1}^{T}(k) \cdots x_{i}^{T}(k) \cdots x_{n}^{T}(k)\right]^{T} \\
u(k)=\left[u_{1}^{T}(k) \cdots u_{i}^{T}(k) \cdots u_{n}^{T}(k)\right]^{T} \\
v(k)=\left[v_{1}^{T}(k) \cdots v_{i}^{T}(k) \cdots v_{n}^{T}(k)\right]^{T} \\
A_{D}=\sum_{i=1}^{n} G_{i} \otimes A_{i i}, \quad B=\sum_{i=1}^{n} G_{i} \otimes B_{i} \\
C=\sum_{i=1}^{n} G_{i} \otimes C_{i}, \quad D=\sum_{i=1}^{n} G_{i} \otimes D_{i}
\end{array}
$$

\section{EXPANSION TO THE HIERARCHICAL OVERLAPPED SYSTEM}

\section{A. Construction of the hierarchical system}

In this first step the hierarchical system $\tilde{S}$ is constructed. The procedure is the following:

1) Overlap initial areas to create bigger ones.

2) Continue with the same procedure after that to create upper level areas until reaching the top layer.

3) Define matrices for expanding system's matrices and variables.

The resulting hierarchical system is presented in Fig. 3.

The state-space model of the hierarchical system is the following (equations (11) and (12)).

$$
\begin{aligned}
& \tilde{x}(k+1)=\tilde{A} \tilde{x}(k)+\tilde{B} \tilde{u}(k)+\tilde{v}(k) \\
& \tilde{z}(k)=\tilde{C} \tilde{x}(k)+\tilde{D} \tilde{u}(k) \\
& \tilde{A} \in \mathcal{A}_{L \tilde{n}}, \tilde{B} \in \mathcal{B}_{L \tilde{n}}, \tilde{C} \in \mathcal{C}_{L \tilde{n}}, \tilde{D} \in \mathcal{D}_{L \tilde{n}}
\end{aligned}
$$

Variables from equations (11) and (12) are the expanded version of the ones from equations (1) and (2). Matrices $\mathcal{A}_{L \tilde{n}}, \mathcal{B}_{L \tilde{n}}, \mathcal{C}_{L \tilde{n}}$ and $\mathcal{D}_{L \tilde{n}}$ are defined according to [9]. Matrices $\tilde{A}, \tilde{B}, \tilde{C}$ and $\tilde{D}$ can be calculated by means of matrices $F(i, \tilde{n}) \in \mathbb{R}^{n_{\tilde{n}} \cdots n_{2} \times n_{\tilde{n}} \cdots n_{2}}$ as shown in equations (14) and (15). $F(i, \tilde{n})$ are responsible of building the new layers by overlapping the system vectors depending, that is, they contain non-zero elements where the variables are defined and zeros elsewhere, in a similar way to $G_{i}(j, k)$ from

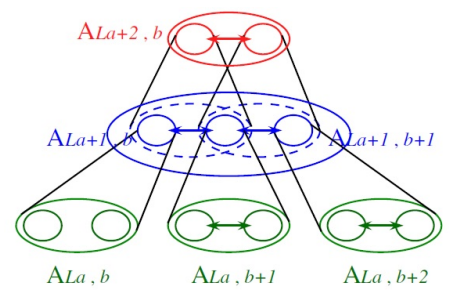

Fig. 3: Hierarchical System [9] equation (10).

$$
\begin{array}{ll}
\tilde{A}=\sum_{i=1}^{n} F(i, \tilde{n}) \otimes A_{i i}, & \tilde{B}=\sum_{i=1}^{n} F(i, \tilde{n}) \otimes B_{i} \\
\tilde{C}=\sum_{i=1}^{n} F(i, \tilde{n}) \otimes C_{i}, & \tilde{D}=\sum_{i=1}^{n} F(i, \tilde{n}) \otimes D_{i}
\end{array}
$$

\section{B. System expansion}

The aim of this second step is verifying the relationship between $\tilde{S}$ and $S$. The conditions for the expansion of the system $S$ to the hierarchical system $\tilde{S}$ are defined on [9]. The expansion matrices are shown in equations (16) to (22).

$$
\begin{array}{rll}
G_{V_{i}} & \in \mathbb{R}^{n_{\tilde{n}} \cdots n_{2} \times n}: G_{V_{i}} G_{j}=0 & \forall i, j, i \neq j \\
G_{U_{i}} & \in \mathbb{R}^{n \times n_{\tilde{n}} \cdots n_{2}}: G_{i} G_{U_{j}}=0 & \forall i, j, i \neq j \\
G_{W_{i}} \in \mathbb{R}^{n \times n_{\tilde{n}} \cdots n_{2}}: G_{i} G_{W_{j}}=0 & \forall i, j, i \neq j
\end{array}
$$

$$
\begin{aligned}
V & =\sum_{i=1}^{n} G_{V_{i}} \otimes I_{n_{1}}: \mathbb{R}^{n \cdot n_{1}} \rightarrow \mathbb{R}^{n_{\tilde{n}} \cdots n_{1}} \\
U_{a} & =\sum_{i=1}^{n} G_{V_{i}} \otimes I_{m_{1}}: \mathbb{R}^{n \cdot m_{1}} \rightarrow \mathbb{R}^{n_{\tilde{n}} \cdots n_{2} m_{1}} \\
U_{b} & =\sum_{i=1}^{n} G_{U_{i}} \otimes I_{m_{1}}: \mathbb{R}^{n_{\tilde{n}} \cdots n_{2} m_{1}} \rightarrow \mathbb{R}^{n \cdot m_{1}} \\
W & =\sum_{i=1}^{n} G_{W_{i}} \otimes I_{p_{1}}: \mathbb{R}^{n_{\tilde{n}} \cdots n_{2} p_{1}} \rightarrow \mathbb{R}^{n \cdot p_{1}}
\end{aligned}
$$

Using matrices defined in equations (16) to (22) the procedure of obtaining the expanded system is defined. The relationships between the expanded system $\tilde{S}$ and the system $S$ are defined as follows (equations (23) to (26)).

$$
\begin{aligned}
& \tilde{x}(k)=V x(k) \\
& \tilde{u}(k)=U_{a} u(k) \\
& u(k)=U_{b} \tilde{u}(k) \\
& z(k)=W \tilde{z}(k)
\end{aligned}
$$

\section{HIERARCHICAL COOPERATIVE MODEL PREDICTIVE CONTROL}

\section{A. Hierarchical control structure}

Once the original system $S$ has been expanded to the hierarchical overlapped system $\tilde{S}$ its control algorithm is designed. The system controlled is the one presented in Fig. 4.

The control procedure steps are as follows:

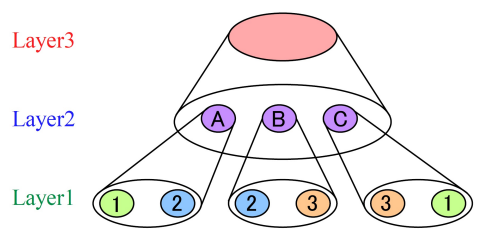

Fig. 4: Hierarchical electrical grid structure 
1) Expand the system from the original one to the hierarchical multilayer one with overlapped subsystems (section III).

2) Compute the economic load dispatch (ELD) for computing the reference values provided by the independent system operator (ISO, explained later in this section).

3) Apply the cooperative model predictive control (LFC) presented in this chapter in following sections to the middle layer (layer 2). Obtain the control inputs to be redirected to the lower layer (layer 3 ).

4) Apply the cooperative model predictive control (LFC) to the lower layer using the information from the previous layer, the middle layer. The result obtained is the control input to be redirected to the original system.

The whole procedure can be seen as an scheme in diagram from Fig. 5.

Both LFC blocks in Fig. 5 include the expansion of the original system to the hierarchical one and the recovery of the original variables after exiting each block.

\section{B. Economic dispatch}

The economic load dispatch (ELD), also known as economic dispatch, is an optimization problem computed by the ISO and generates the reference power values for the electrical power network. The problem, based on the one from [6], is stated in equations (27) to (28).

$$
\begin{aligned}
\min _{P_{G_{i j}}} & \sum_{i=1}^{M} \sum_{j=1}^{2}\left(a_{g, i j} P_{G_{i j}}^{2}+b_{g, i j} P_{G_{i j}}+c_{g, i j}\right) \\
\text { Subject to } & \sum_{j=1}^{2} P_{G_{i j}}+P_{t i e_{i}}+P_{W_{i}}-P_{L_{i}}+P_{B_{i}}=0 \\
& \sum_{j=1}^{M} P_{t i e_{i}}=0 \\
& P_{G_{i j}}^{\min } \leq P_{G_{i j}} \leq P_{G_{i j}}^{\max } \\
& \Delta P_{G_{i j}}^{\min } \leq \Delta P_{G_{i j}} \leq \Delta P_{G_{i j}}^{\max } \\
& P_{t i e_{i}}^{\min _{i n}} \leq P_{t i e_{i}} \leq P_{t i e_{i}}^{\max }
\end{aligned}
$$

\section{Predictive model}

To be able to apply load frequency control (LFC) based on MPC, the corresponding predictive model for each area $i$

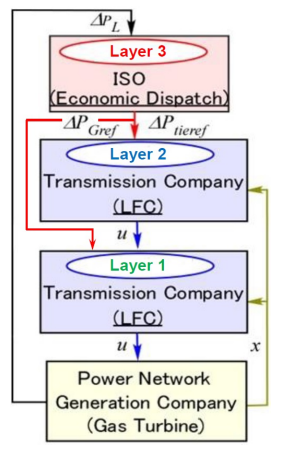

Fig. 5: System diagram needs to be built. A prediction horizon $N$ in order to define future predicted values. The number of areas of the system is $M$ and the model is presented in equations (29) to (33).

$$
\bar{x}_{i}=\bar{A}_{i}(k)+\sum_{j=1, j \neq i}^{M}\left(\bar{A}_{i j}^{0} x_{j}(k)+\bar{A}_{i j} \bar{x}_{j}\right)+\bar{B}_{i} \bar{u}_{i}+\bar{A}_{d i} \bar{d}_{i}
$$

Providing that,

$$
\begin{aligned}
\bar{x}_{i} & =\left[x_{i}^{T}(k+1) \cdots x_{i}^{T}(k+N)\right]^{T} \\
\bar{x}_{j} & =\left[x_{j}^{T}(k+1) \cdots x_{j}^{T}(k+N)\right]^{T} \\
\bar{u}_{i} & =\left[u_{i}^{T}(k) \cdots u_{i}^{T}(k+N-1)\right]^{T} \\
\bar{d}_{i} & =\left[u_{i}^{T}(k) \cdots u_{i}^{T}(k+N-1)\right]^{T}
\end{aligned}
$$

Previous models for each area are assembled to obtain the state-space representation for the whole system. The result is the one in equations (34) to (42).

$$
\mathcal{A} \bar{x}=\overline{\mathcal{A}} \bar{x}(k)+\overline{\mathcal{B}} \bar{u}+\overline{\mathcal{A}}_{d} \bar{d}
$$

Providing that,

$$
\begin{aligned}
& \mathcal{A}=\left[\begin{array}{cccc}
I & -A_{12} & \ldots & -A_{1 M} \\
-A_{21} & I & \ldots & -A_{2 M} \\
\vdots & \vdots & \ddots & \vdots \\
-A_{M 1} & A_{M 2} & \ldots & I
\end{array}\right] \\
& \overline{\mathcal{A}}=\left[\begin{array}{cccc}
\bar{A}_{1} & \bar{A}_{12}^{0} & \ldots & \bar{A}_{1 M}^{0} \\
\bar{A}_{21}^{0} & \bar{A}_{2}^{0} & \ldots & \bar{A}_{2 M}^{0} \\
\vdots & \vdots & \ddots & \vdots \\
\bar{A}_{M 1}^{0} & \bar{A}_{M 2}^{0} & \ldots & \bar{A}_{M}^{0}
\end{array}\right] \\
& \overline{\mathcal{B}}=\left[\begin{array}{cccc}
\bar{B}_{1} & 0 & \ldots & 0 \\
0 & \bar{B}_{2} & \ldots & 0 \\
\vdots & \vdots & \ddots & \vdots \\
0 & 0 & \ldots & \bar{B}_{M}
\end{array}\right] \\
& \overline{\mathcal{A}}_{d}=\left[\begin{array}{cccc}
\bar{A}_{d 1} & 0 & \ldots & 0 \\
0 & \bar{A}_{d 2} & \ldots & 0 \\
\vdots & \vdots & \ddots & \vdots \\
0 & 0 & \ldots & \bar{A}_{d M}
\end{array}\right] \\
& \bar{x}=\left[\bar{x}_{1}^{T} \cdots \bar{x}_{i}^{T} \cdots \bar{x}_{i}^{T}\right]^{T} \\
& \bar{x}(k)=\left[\bar{x}_{1}^{T}(k) \cdots \bar{x}_{i}^{T}(k) \cdots \bar{x}_{i}^{T}(k)\right]^{T} \\
& \bar{u}=\left[\bar{u}_{1}^{T} \cdots \bar{u}_{i}^{T} \cdots \bar{u}_{i}^{T}\right]^{T} \\
& \bar{d}=\left[\bar{d}_{1}^{T} \cdots \bar{d}_{i}^{T} \cdots \bar{d}_{i}^{T}\right]^{T}
\end{aligned}
$$

Future disturbances are predicted as follows, where $T$ is the sampling time and $T_{r e f}$ a reference time.

$$
\begin{aligned}
d_{i}(k) & =d_{i}(k) \\
d_{i}(k+1) & =d_{i}(k) e^{-1 * \frac{T}{T_{r e f}}} \\
\vdots & \\
d_{i}(k+N) & =d_{i}(k) e^{-N * \frac{T}{T_{r e f}}}
\end{aligned}
$$


A similar procedure is the one used for predicting future values of the systems' reference states. The procedure continues by multiplying both sides of equation (34) by the inverse of the left-hand-side matrix. After doing so the resulting system is the one presented in equation (43).

$$
\begin{gathered}
\bar{x}=\overline{\mathcal{A}}^{\prime} \bar{x}(k)+\overline{\mathcal{B}}^{\prime} \bar{u}+\overline{\mathcal{A}}_{d}^{\prime} \bar{d} \\
\overline{\mathcal{A}}^{\prime}=\mathcal{A}^{-1} \overline{\mathcal{A}}, \quad \overline{\mathcal{B}}^{\prime}=\mathcal{A}^{-1} \overline{\mathcal{B}}, \quad \overline{\mathcal{A}}_{d}^{\prime}=\mathcal{A}^{-1} \overline{\mathcal{A}}_{d}
\end{gathered}
$$

Manipulating matrices presented in equations (43) to (44) a definitive state-space model for each area can be obtained. The result is presented in equations (45) to (46).

$$
\begin{gathered}
\bar{x}_{i}=\bar{A}_{i}^{\prime} x_{i}(k)+\bar{B}_{i}^{\prime} \bar{u}_{i}+\bar{A}_{d i}^{\prime} \bar{d}_{i} \\
+\sum_{j=1, j \neq i}^{M}\left(\bar{A}_{i j}^{\prime} \bar{x}_{j}(k)+\bar{B}_{i j}^{\prime} \bar{u}_{j}+\bar{A}_{d i j}^{\prime} \bar{d}_{j}\right) \\
\bar{A}_{i}^{\prime}=\mathcal{A}_{i i}^{-1} \bar{A}_{i}, \quad \bar{A}_{i j}^{\prime}=\overline{\mathcal{A}}_{i j}^{-1} \bar{A}_{j i}^{0} \\
\bar{B}_{i}^{\prime}=\mathcal{A}_{i i}^{-1} \bar{B}_{i}, \quad \bar{B}_{i j}^{\prime}=\overline{\mathcal{A}}_{i j}^{-1} \bar{B}_{j} \\
\bar{A}_{d i}^{\prime}=\mathcal{A}_{i i}^{-1} \bar{A}_{d i}, \quad \bar{A}_{d i j}^{\prime}=\overline{\mathcal{A}}_{i j}^{-1} \bar{A}_{d j}
\end{gathered}
$$

Values of $\mathcal{A}_{i j}^{-1}$ are those of the $(i, j)$ components of the $\mathcal{A}^{-1}$ matrix. As it has been said, $\mathcal{A}_{i j}^{-1}$ is the inverse matrix of $\mathcal{A}_{i j}$.

\section{Cooperative model predictive control}

The system presented is the lower level of the hierarchical system, which is regulated by the load frequency control (LFC) is implemented based on the procedure in [10], using appropriate diagonal weighting matrices $Q_{i}$ and $R_{i}$. To be able to implement the MPC for this level the follow optimization problem must be solved for every system iteration in order to compute the successive control inputs $u_{i}^{p *}$.

$$
\begin{aligned}
u_{i}^{p *} & =\operatorname{argmin}_{u_{i}} \quad \Phi_{i}\left(u_{1}^{p-1} u_{2}^{p-1} \cdots u_{i}^{p-1} u_{M}^{p-1}: x_{i}(k)\right) \\
& =\operatorname{argmin}_{u_{i}} \quad u_{i}^{T} \mathcal{R} u_{i}+2\left(r_{i}(x)+h_{i}(d)\right. \\
& \left.+\mathcal{X}_{i}\left(x^{\text {ref }}\right) \sum_{j=1, j \neq i}^{M} \mathcal{B}_{i j} u_{j}^{p-1}\right) u_{i}+\text { const. }
\end{aligned}
$$

Providing that,

$$
\begin{aligned}
\mathcal{R} & =R_{i}+\bar{B}_{i}^{\prime T} Q_{i} \bar{B}_{i}^{\prime}+\sum_{j=1, j \neq i}^{M} \bar{B}_{j i}^{\prime T} Q_{j} \bar{B}_{j i}^{\prime} \\
r_{i}(x) & =\bar{B}_{i}^{\prime T} Q_{i} \bar{A}_{i}^{\prime} x_{i}(k)+\sum_{j=1, j \neq i}^{M} \bar{B}_{j i}^{\prime} Q_{j} \bar{A}_{i}^{\prime} x_{i}(k) \\
& +\sum_{j=1, j \neq i}^{M} \sum_{l=1, l \neq i}^{M} \bar{B}_{j i}^{\prime T} Q_{j} \bar{A}_{i l}^{\prime} x_{l}(k) \\
h_{i}(d) & =\bar{B}_{i}^{\prime T} Q_{i} \bar{A}_{d i}^{\prime} d_{i}+\sum_{j=1, j \neq i}^{M} \bar{B}_{j i}^{\prime T} Q_{j} \bar{A}_{d i}^{\prime} d_{i} \\
& +\sum_{j=1, j \neq i}^{M} \sum_{l=1, l \neq i}^{M} \bar{B}_{j i}^{\prime T} Q_{j} \bar{A}_{d i l}^{\prime} d_{l}
\end{aligned}
$$

$$
\begin{aligned}
\mathcal{X}_{i}\left(x^{r e f}\right) & =x_{i}^{r e f(T)} Q_{i} \bar{B}_{i}+\sum_{j=1, j \neq i}^{M}+x_{j}^{r e f(T)} Q_{j} \bar{B}_{j i} \\
\mathcal{B}_{i j} & =\bar{B}_{i}^{\prime} T Q_{i} \bar{B}_{i j}^{\prime}+\bar{B}_{j i}^{\prime} T Q_{j} \bar{B}_{j}^{\prime}+\sum_{l=1, l \neq i, j}^{M} \bar{B}_{l i}^{\prime T} Q_{l} \bar{B}_{l j}^{\prime}
\end{aligned}
$$

Subject to,

$$
\begin{aligned}
& \bar{x}_{i}^{\text {min }} \leq \bar{x}_{i}(k+j) \leq \bar{x}_{i}^{\max } \\
& \bar{u}_{i}^{\text {min }} \leq \bar{u}_{i}(k+j) \leq \bar{u}_{i}^{\text {max }} \\
& \bar{u}_{i}(k+j \mid k)=0 \quad\left(j \geq H_{u}\right)
\end{aligned}
$$

In the previous optimization problem the variables are the ones in equation (48).

$$
\begin{aligned}
d_{i} & =\bar{d}_{i}=\left[d_{i}^{T}(k) \cdots d_{i}^{T}(k+N-1)\right]^{T} \\
x_{i}^{r e f} & =\bar{x}_{i}^{r e f}=\left[x_{i}^{r e f(T)}(k+1) \cdots x_{i}^{r e f(T)}(k+N)\right]^{T}
\end{aligned}
$$

- $x_{i}(k)$ are the state variables at sampling time $\mathrm{k}$.

- $d_{i}$ are the $N$ predicted values of the disturbance variables.

- $x_{i}^{r e f}$ are the $N$ predicted reference values of the state variables.

\section{SIMULATION}

\section{A. Simulation conditions}

Once the control scheme has been described, simulations have been designed using Matlab $\mathrm{R} /$ Simulink $\mathrm{R}$. The simulated number of areas is $M=3$ while the prediction horizon is $N=5$ and the control horizon is $H_{u}=1$. System matrices for each area in its continuous form are as follows (equations (52) to (62)).

$$
\begin{aligned}
& x(t)= {\left[x_{1}^{T}(t) x_{2}^{T}(t) x_{3}^{T}(t)\right]^{T} } \\
& {\left[\begin{array}{c}
\frac{1}{M_{i}} \\
\Delta f_{i}(t) \\
\Delta P_{G_{i}}(t) \\
\Delta x_{g_{i}}(t) \\
\Delta P_{B_{i}}(t) \\
\Delta P_{H_{i}}(t) \\
U_{i}(t) \\
\Delta P_{t i e_{i}}(t)
\end{array}\right] \in \mathbb{R}^{7 \cdot 3} } \\
& x_{i}(t)= {\left[u_{1}^{T}(t) u_{2}^{T}(t) u_{3}^{T}(t)\right]^{T} \in \mathbb{R}^{3 \cdot 3} } \\
& u_{i}(t) \\
& u_{i}(t)=\left[\Delta u_{g_{i}}(t) \Delta u_{B_{i}}(t) \Delta u_{H_{i}}(t)\right]^{T} \\
& w(t)=\left[w_{1}^{T}(t) w_{2}^{T}(t) w_{3}^{T}(t)\right]^{T} \in \mathbb{R}^{1 \cdot 3} \\
& w_{i}(t)=\left[\Delta P_{W_{i}}(t)-\Delta P_{L_{i}}(t)\right]^{T} \\
& z(t)=\left[z_{1}^{T}(t) z_{2}^{T}(t) z_{3}^{T}(t)\right]^{T} \in \mathbb{R}^{10 \cdot 3}
\end{aligned}
$$




$$
\begin{aligned}
& A_{\text {cii }}= \\
& {\left[\begin{array}{ccccccc}
-\frac{D_{i}}{M_{i}} & \frac{1}{M_{i}} & 0 & \frac{1}{M_{i}} & -\frac{1}{M_{i}} & 0 & \frac{1}{M_{i}} \\
0 & -\frac{1}{T_{G i}} & \frac{1}{T_{G i}} & 0 & 0 & 0 & 0 \\
-\frac{1}{T_{g i} R_{g i}} & 0 & -\frac{1}{T_{g i}} & 0 & 0 & 0 & 0 \\
0 & 0 & 0 & -\frac{1}{T_{B i}} & 0 & 0 & 0 \\
0 & 0 & 0 & 0 & -\frac{1}{T_{H i}} & 0 & 0 \\
-k_{i} & 0 & 0 & 0 & 0 & 0 & 1 \\
a_{71} & 0 & 0 & 0 & 0 & 0 & 0
\end{array}\right]} \\
& a_{71}=-\sum_{j \in \mathcal{N}_{i}} T_{i j} \\
& A_{c i j}=\operatorname{diag}\left(T_{i j}, 0,0,0,0,0,0\right), T_{i j}=0 \text { if }(i, j) \notin \mathcal{N} \\
& B_{c i}=\left[\begin{array}{ccc}
0 & 0 & 0 \\
0 & 0 & 0 \\
\frac{1}{T_{g i}} & 0 & 0 \\
0 & \frac{1}{T_{B i}} & 0 \\
0 & 0 & \frac{1}{T_{H i}} \\
0 & 0 & 0 \\
0 & 0 & 0
\end{array}\right], H_{c i}=\left[\begin{array}{c}
\frac{1}{M_{i}} \\
0 \\
0 \\
0 \\
0 \\
0 \\
0
\end{array}\right] \\
& C_{c i}=\left[\begin{array}{ccccccc}
10 & 0 & 0 & 0 & 0 & 0 & 0 \\
0 & 1 & 0 & 0 & 0 & 0 & 0 \\
0 & 0 & 1 & 0 & 0 & 0 & 0 \\
0 & 0 & 0 & 1 & 0 & 0 & 0 \\
0 & 0 & 0 & 0 & 1 & 0 & 0 \\
0 & 0 & 0 & 0 & 0 & 1 & 0 \\
0 & 0 & 0 & 0 & 0 & 0 & 1 \\
0 & 0 & 0 & 0 & 0 & 0 & 0 \\
0 & 0 & 0 & 0 & 0 & 0 & 0 \\
0 & 0 & 0 & 0 & 0 & 0 & 0
\end{array}\right], D_{c i}=\left[\begin{array}{ccc}
0 & 0 & 0 \\
0 & 0 & 0 \\
0 & 0 & 0 \\
0 & 0 & 0 \\
0 & 0 & 0 \\
0 & 0 & 0 \\
0 & 0 & 0 \\
1 & 0 & 0 \\
0 & 10 & 0 \\
0 & 0 & 10
\end{array}\right]
\end{aligned}
$$

The meaning of the state variables presented in equation (53) is as follows:

- $\Delta f_{i}$, frequency deviation in area $i$.

- $\Delta P_{G_{i}}$, gas turbine output power deviation in area $i$.

- $\Delta x_{g_{i}}$, governor input deviation in area $i$.

- $\Delta P_{B_{i}}$, battery system output power deviation in area $i$.

- $\Delta P_{H_{i}}$, heat pump output power deviation in area $i$.

- $U_{i}=\int A R_{i} d t=\int\left(\Delta P_{t i e_{i}}-k_{i} \Delta f_{i}\right) d t$

- $\Delta P_{t i e_{i}}$, tie-line power deviation in area $i$.

Control input variables in equation (53) are:

- $\Delta u_{g_{i}}$, gas turbine control input deviation in area $i$.

- $\Delta u_{B_{i}}$, battery system control input deviation in area $i$.

- $\Delta u_{H_{i}}$, heat pump control input deviation in area $i$.

Finally, disturbances for each area $i$ presented in equation (57) are composed by the wind power deviation $\Delta P_{W_{i}}$ and the load power deviation $\Delta P_{L_{i}}$.

Regarding the cost optimization coefficients of the electrical grid, these are the ones shown in Table I. Similarly, electrical grid parameters and their meaning are presented in Table II.

The total amount of economic load dispatch (ELD) iterations has been set to 8 and, for the case of load frequency control (LFC), to 24. Wind power and load disturbances are
TABLE I: ELD parameters

\begin{tabular}{|c|ccc|}
\hline$a_{g, i j}$ & $1 \times 10^{5}$ & $1.2 \times 10^{5}$ & $8 \times 10^{4}$ \\
\hline$b_{g, i j}$ & $3.3 \times 10^{3}$ & $3.3 \times 10^{3}$ & $3.33 \times 10^{3}$ \\
\hline$c_{g, i j}$ & $3.3 \times 10^{-4}$ & $3.3 \times 10^{-4}$ & $3.3 \times 10^{-4}$ \\
\hline
\end{tabular}

TABLE II: System parameters

\begin{tabular}{|l|c|lll|}
\hline Sampling time [s] & $T$ & \multicolumn{4}{|c|}{0.1} \\
\hline ISO Sampling time [s] & $T_{I S O}$ & \multicolumn{4}{|c|}{150} \\
\hline Local Sampling time [s] & $T_{L o c a l}$ & \multicolumn{4}{|c|}{50} \\
\hline Reference frequency [Hz] & $f$ & \multicolumn{4}{|c|}{50} \\
\hline System capacity [MW] & $S_{i}$ & 50 & 50 & 50 \\
\hline Inertia constant [puMWs/Hz] & $M_{i}$ & 0.21 & 0.22 & 0.20 \\
\hline Damping constant [puMW/Hz] & $D_{i}$ & 0.25 & 0.24 & 0.26 \\
\hline Gas turbine time constant [s] & $T_{G_{i}}$ & \multicolumn{5}{|c|}{5} & 5 & 5 \\
\hline Governor time constant [s] & $T_{g_{i}}$ & 0.2 & 0.2 & 0.2 \\
\hline Spin ratio [Hz/puMW] & $R_{g_{i}}$ & 2.5 & 2.5 & 2.5 \\
\hline Battery inverter time constant [s] & $T_{B_{i}}$ & \multicolumn{4}{|c|}{1} & 1 & 1 \\
\hline Heat pump time constant [s] & $T_{H_{i}}$ & \multicolumn{4}{|c|}{1} & 1 & 1 \\
\hline Tie cable constant [puMW] & $T_{t i e}$ & \multicolumn{4}{|c|}{0.5} \\
\hline
\end{tabular}

defined to be able to analyse the system behaviour under several conditions. These disturbances are as follows (Fig. 6 and 7).

\section{B. Simulation results}

1) Grid frequency control: The main target of this study is maintaining the frequency as constant as possible. Frequency deviation for each area using the control algorithm proposed are seen in Fig. 8 while the ones obtained in [9] are presented in Fig. 9. The fact that the model used includes the same parameters as [9] makes the analysis quite straightforward.

It can be seen that both areas 1 and 3 in Fig. 8 have a resulting frequency deviation lower than the one obtained in Fig. 9. Area 2 presents a singularity around second 1000 , when the disturbances from Fig. 7 evolve differently, but this

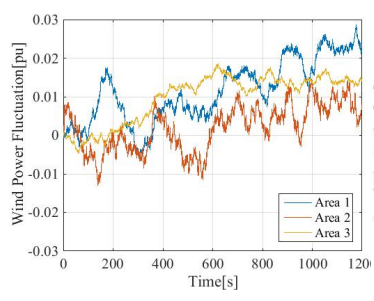

Fig. 6: Wind power disturbance
Fig. 7: Load disturbance
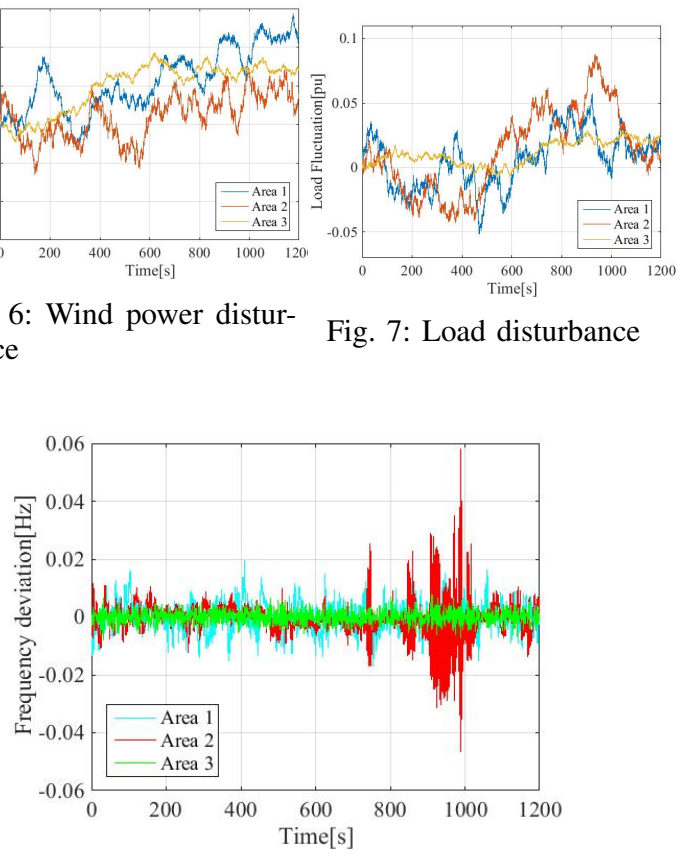

Fig. 8: Frequency deviation for all areas (proposed) 


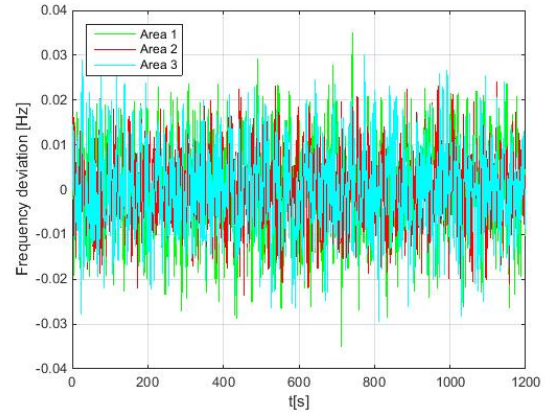

Fig. 9: Frequency deviation for all areas (obtained in [9])

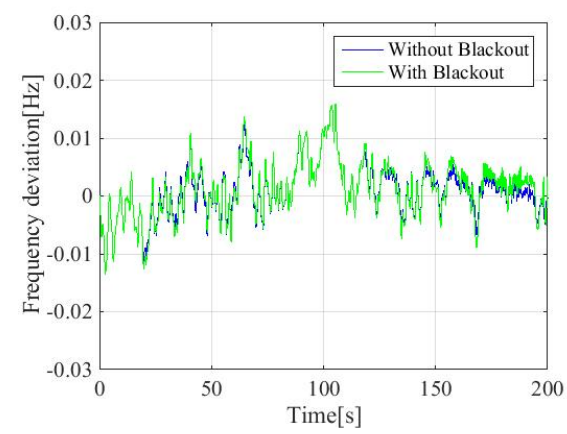

Fig. 10: Frequency deviation comparison with blackout until $\mathrm{t}=10 \mathrm{~s}$

singularity reaches a maximum value of $0.06 \mathrm{~Hz}$ (lower than the maximum allowed deviation of $\pm 0.2 \mathrm{~Hz}$ ) and goes back to average values around $0.05 \mathrm{~Hz}$ after 100 seconds.

2) Control during communication blackouts: The hierarchical cooperative Model Predictive Control presented makes the system quite resistant to eventual communication blackouts in certain areas. As an example, a blackout in area 1 during the first 10 seconds has been simulated. The frequency deviation of the first area is compared in both cases, with or without blackout. The results can be seen in Fig. 10.

It should be noted that the control input is generated even during the communication blackout because of its relationship with the other subsystems and the frequency deviation remains low enough. It can be seen that both curves with and without blackout the frequency deviation is quite similar. At the beginning both signals are even indistinguishable.

\section{CONCLUSIONS}

The study presented is based on using distributed cooperative model predictive control applied to an electrical grid. The first step has been defining the network system state-space model and after that a hierarchical version of that model has been constructed. The hierarchical version of the system is layered in order to ensure control in case of hypothetical blackouts in some areas.

Once the system model has been expanded, a multilevel control has been applied. Reference values coming from the upper layer have been computed by using an economic load dispatch (ELD) consisting on a cost minimization. Lower layers have been controlled using these reference values provided by the ELD on a load frequency control (LFC) for each layer. The resulting control inputs computed by each layer are used for lower layers in order to obtain a better result. This combination of cooperative model predictive control and multilayer hierarchical control with internal feedback loops is what makes this study different from the references consulted, such as [9] and [7]. Simulations for implementing the system and related algorithms have been designed using Matlab@/Simulink(R).

According to the main objectives, frequency deviation is kept, except for certain sudden peaks (caused by exceeding values of the disturbances signals defined) which disappear quite rapidly, to levels which are lower than the ones in [9], as seen in the simulation results. It has also been seen that the system reacts accordingly when a communication blackout affects one area. The other areas are able to provide a control signal to the disconnected area. Finally, the system cost has been kept around reasonable values. Further research in implementing other methods of Economic Dispatch such as the one in [11] is an option to be done for the future.

\section{ACKNOWLEDGMENT}

This work has been supported by the project DPI201569286-C3-2-R of the Spanish Ministerio de Educación de España and 2014 SGR 267 of the AGAUR agency of the Generalitat de Catalunya.

\section{REFERENCES}

[1] X. Zhou, T. Huang, Y. Ma, Z. Gao, "The Research on Smart Distribution Grid". International Conference on Mechatronics and Automation. Aug. 7-10 in Harbin, China. 2016.

[2] X. Zhang, A. Papachristodoulou, N. Li, "Distributed Optimal Steadystate Control Using Reverse- and Forward-engineering". 2015 IEEE 54th Annual Conference on Decision and Control (CDC). Dec. 15-18 in Osaka, Japan. 2015.

[3] X. Zhang, N. Li, A. Papachristodoulou, "Achieving Real-time Economic Dispatch in Power Networks via a Saddle Point Design Approach". 2015 IEEE Power and Energy Society (PES) General Meeting Conference. July 26-30 in Denver, Colorado, USA. 2015.

[4] J. B. Rawlings, D. Q. Mayne, "Model Predictive Control: Theory and Design", Nob Hill Publishing. 2009.

[5] A. M. Ersdal, L. Imsland, K. Uhlen, "Model Predictive LoadFrequency Control". IEEE Transactions on Power Systems. Vol.31. Issued Jan. 1. 2016.

[6] F. Berkel, D. Gorges, S. Liu, "Load-frequency control, economic dispatch and unit commitment in smart microgrids based on hierarchical model predictive control". IEEE 52nd Conference on Decision and Control, pp. 2326-2333, 2013.

[7] A. N. Venkat, I. A. Hiskens, J. B. Rawlings, S. J. Wright, "Distributed MPC Strategies With Application to Power System Automatic Generation Control". IEEE Transactions on Control Systems Technology, vol. 16, no. 6, pp. 1192-1206. 2008.

[8] M. Ikeda, D. D. Šiljak, D. E. White, "Decentralized Control with Overlapping Information Sets". Journal of optimization theory and applications, Vol.34, No.2, June 1981.

[9] T. Suehiro, T. Namerikawa, "Hierarchical Control of Power Networks by using Overlapping Information". 14th International Conference on Control, Automation and Systems(ICCAS 2014). Oct. 22-25, 2014 in KINTEX, Gyeonggi-do, Korea. 2014.

[10] S. Takamura, T. Namerikawa, "Hierarchical and Cooperative Model Predictive Control for Load Frequency of Power Network". 35th Chinese Control Conference (CCC2016). July 27-29, 2016 in Chengdu, China. 2016.

[11] S. Yang, S. Tan, J. Xu, "Consensus Based Approach for Economic Dispatch Problem in a Smart Grid". IEEE Transactions on Power Systems. Vol.28. Issued Nov. 4. 2013. 